\section{Impact of timely antibiotic administration on outcomes in patients with severe sepsis and septic shock in the emergency department}

\author{
Young Min Joo, Minjung Kathy Chae, Sung Yeon Hwang, \\ Sang-Chan Jin, Tae Rim Lee, Won Chul Cha, Ik Joon Jo, Min Seob Sim, \\ Keun Jeong Song, Yeon Kwon Jeong, Tae Gun Shin
}

Department of Emergency Medicine, Samsung Medical Center, Sungkyunkwan University School of Medicine, Seoul, Korea

Objective We aimed to investigate the effect of timely antibiotic administration on outcomes in patients with severe sepsis and septic shock.

Methods We analyzed data from a sepsis registry that included adult patients who initially presented to the emergency department (ED) and met criteria for severe sepsis or septic shock. Timely antibiotic use was defined as administration of a broad-spectrum antibiotic within three hours from the time of ED arrival. Multivariable logistic and linear regression analyses were performed to assess associations between timely administration of antibiotics and outcomes, including hospital mortality, 48-hour change in Sequential Organ Failure Assessment (SOFA) score (delta SOFA), and hospital length of stay (LOS).

Results A total of 591 patients were included in the study. In-hospital mortality was $16.9 \%$ for patients receiving timely antibiotics $(n=377)$ and $22.9 \%$ for patients receiving delayed antibiotics $(n=214 ; P=0.04)$. The adjusted odds ratio for in-hospital survival was $0.54(95 \%$ confidence interval $[\mathrm{CI}], 0.34$ to $0.87 ; \mathrm{P}=0.01$ ) in patients who received timely antibiotics. Timely antibiotic administration was also significantly associated with higher delta SOFA (2 vs. 1) and shorter hospital LOS among survivors (11 days vs. 15 days). Multivariable linear regression analyses showed that timely antibiotic administration was significantly associated with increased delta SOFA and decreased hospital LOS.

Conclusion Antibiotic administration within three hours from the time of ED arrival was significantly associated with improved outcomes, including in-hospital survival, reversal of organ failure, and shorter hospital LOS, in patients with severe sepsis and septic shock.

Keywords Anti-bacterial agents; Sepsis; Mortality; Multiple organ failure

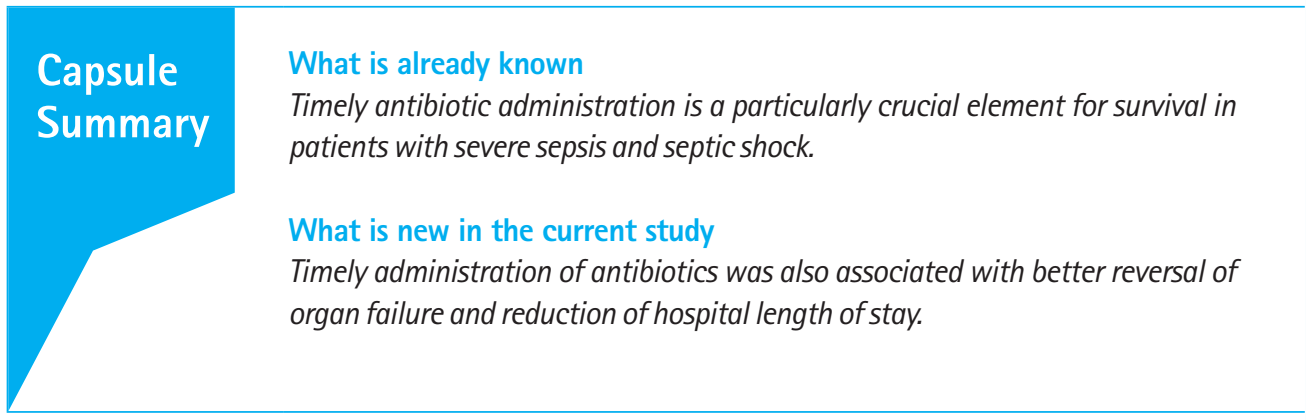

elSSN: 2383-4625

Received: 27 June 2014

Revised: 8 August 2014

Accepted: 12 August 2014

Correspondence to: Tae Gun Shin Department of Emergency Medicine, Samsung Medical Center,

Sungkyunkwan University School of Medicine, 81 Irwon-ro, Gangnam-gu, Seoul 135-710, Korea

E-mail: tackles@naver.com

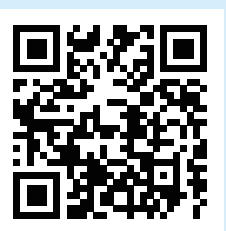

How to cite this article:

Joo YM, Chae MK, Hwang SY, Jin SC, Lee TR, Cha WC, Jo IJ, Sim MS, Song KJ, Jeong YK, Shin TG. Impact of timely antibiotic administration on outcomes in patients with severe sepsis and septic shock in the emergency department. Clin Exp Emerg Med 2014;1(1):35-40.

This is an Open Access article distributed under the terms of the Creative Commons Attribution Non-Commercial License (http:// creativecommons.org/licenses/by-nc/3.0/). 


\section{INTRODUCTION}

Severe sepsis and septic shock are severe illnesses caused by infection that can lead to death through progression of systemic inflammatory response syndrome and multiple organ failure. ${ }^{1,2}$ Mortality rates are reported to range from 20\% to 50\%, and with the recent steady rise in incidence rates, there is increasing emphasis on early and active treatment of sepsis. ${ }^{3-5}$

Proper treatment of the infection source is vital for effective and prompt treatment of patients with severe sepsis and septic shock. Early antibiotic treatment is a particularly crucial element. ${ }^{6}$ In patients with septic shock, a delay in antibiotic administration from the time when hypotension first appears is significantly associated with increased mortality rates, and similar results have been reported in patients with severe sepsis. ${ }^{7-9}$ Based on existing observational studies, the 2012 Surviving Sepsis Campaign (SSC) guidelines recommend antibiotic administration within one hour of diagnosis of sepsis or prompt antibiotic administration within 3 hours for patients in the emergency department (ED). ${ }^{10}$

This study explored the differences in prognosis based on early administration of antibiotics as recommended by the SSC guidelines in ED patients with severe sepsis and septic shock. We investigated the effect of early antibiotic administration on outcomes by assessing in-hospital mortality, hospital length of stay (LOS), and recovery from organ failure.

\section{METHODS}

Using a retrospective cohort study design, we analyzed data from a sepsis registry comprised of patients who presented to the ED of Samsung Medical Center, an urban tertiary teaching hospital with 70,000 visits per year. The Institutional Review Board approved this study, and informed consent was waived because the study was retrospective and no intervention was required.

\section{Patient inclusion criteria}

The subjects of this study were patients over 18 years of age with severe sepsis who had initial blood lactate concentrations of over $4 \mathrm{mmol} / \mathrm{L}$ and septic shock diagnosed at the time of ED arrival between August 2008 and March 2012. We excluded patients with terminal malignancies or a previously signed "Do Not Attempt Resuscitation (DNAR)" order, as well as patients who refused early goal-directed therapy.

\section{Definitions}

Sepsis was defined as suspected or confirmed infection in the presence of two or more systemic inflammatory response syndrome criteria. The systemic inflammatory response syndrome is defined by two or more of the following conditions: (1) body temperature greater than $38^{\circ} \mathrm{C}$ or less than $36^{\circ} \mathrm{C}_{i}(2)$ heart rate greater than 90 beats per minute; (3) respiratory rate greater than 20 breaths per minute or $\mathrm{PaCO}_{2}$ of less than $32 \mathrm{mmHg}$; and (4) white blood cell count greater than $12,000 / \mathrm{mm}^{3}$, less than $4,000 / \mathrm{mm}^{3}$, or the presence of more than 10\% immature neutrophils ("bands"). ' Severe sepsis was defined as sepsis associated with acute organ dysfunction. Septic shock was defined as sepsis that presented with hypotension (systolic blood pressure $<90 \mathrm{mmHg}$, mean arterial pressure [MAP] $<60 \mathrm{mmHg}$, or a reduction in systolic blood pressure of $>40 \mathrm{mmHg}$ from baseline) despite adequate fluid resuscitation, in the absence of other causes for hypotension. ${ }^{2}$

Early antibiotic use was defined as administration of a broadspectrum antibiotic within three hours from the time of ED arrival. ${ }^{11}$ All patients were classified into either the early administration group or delayed administration group for comparison.

\section{Data collection}

We analyzed the sepsis registry, which had been prospectively collected since August of 2008, for relevant patients presenting to the ED. ${ }^{12}$ During the study period, we recommended and monitored delivery of the resuscitation bundle for patients with severe sepsis or septic shock based on the protocol by Rivers et al. ${ }^{13}$ and the 2008 SSC guidelines."

For intergroup comparisons, data included detailed patient characteristics, comorbidities, vital signs, sites of infection, hemodynamic indices, laboratory data, use of vasopressors, and use of mechanical ventilation. Data about achievements of early resuscitation targets were also recorded, including MAP $\geq 65 \mathrm{mmHg}$, central venous pressure (CVP) $\geq 8 \mathrm{mmHg}$, and central venous oxygen saturation $\left(\mathrm{ScvO}_{2}\right) \geq 70 \%$. We assessed the achievement of resuscitation targets in six hours from the time zero, which was defined as when either hypotension or hyperlactatemia greater than $4 \mathrm{mmol} / \mathrm{L}$ was reported. If central line insertion followed by measurement of $\mathrm{ScvO}_{2}$ was not performed, the targets of CVP and $\mathrm{ScvO}_{2}$ were considered as not achieved. Acute Physiology and Chronic Health Evaluation (APACHE) II scores were examined for assessment of severity, ${ }_{1}^{14}$ and Sequential Organ Failure Assessment (SOFA) scores were examined at the time of diagnosis and 48 hours later for assessment of organ dysfunction. 15,16

\section{Outcome measures}

In-hospital mortality was the primary endpoint, and the secondary endpoints were 48-hour change in SOFA score, which reflected recovery from organ failure (delta SOFA $=$ SOFA at ED recognition - SOFA after 48 hours); hospital LOS; intensive care unit (ICU) 
admission rate; and length of ICU stay. ${ }^{15}$

\section{Statistical analysis}

Continuous variables were presented as the median and interquartile range (IQR), and the Wilcoxon rank sum test was used for comparisons. Categorical variables were compared using the chi-squared test. For analysis of the association with the in-hospital mortality rate, potential confounding variables were introduced in a backward stepwise logistic regression model (variable exit threshold set at $P>0.05$ ) including demographic factors (age, comorbidities, sites of infection), severity factors (APACHE II score, initial blood lactate concentration), and treatment factors (achievement of early resuscitation targets). The Hosmer-Lemeshow test was used to check the goodness of fit of the logistic regression. Correlation analyses were performed to evaluate the impacts of early antibiotic administration on delta SOFA and hospital LOS of survivors through multivariable linear regression analysis with backward stepwise procedures as above. Hospital LOS was analyzed after log transformation to achieve normality for linear regression analysis. A P-value less than 0.05 was considered significant. STATA ver. 11.0 (STATA Co., College Station, TX USA) was used for statistical analysis.

\section{RESULTS}

\section{Baseline characteristics}

Among a total of 738 patients with severe sepsis and septic shock, 591 patients were analyzed (Table 1). The excluded patients included 116 terminal cancer patients, 27 DNAR patients, and 4 patients who rejected early goal-directed therapy. Median age for the overall cohort was 66 years (IQR, 55 to 73 years) and there were 330 men (55.8\%). The in-hospital mortality rate for all patients was $18.6 \%$. The number of patients who received early antibiotic administration was 377 patients (63.8\%), and

Table 1. Comparison of baseline patient characteristics

\begin{tabular}{|c|c|c|c|c|}
\hline Variable & All patients $(n=591)$ & Early group $(n=377)$ & Delayed group $(n=214)$ & P-value \\
\hline Age (yr) & $66(55-73)$ & $65(55-73)$ & $67(57-73)$ & 0.46 \\
\hline Gender (male) & $330(55.8)$ & $214(56.8)$ & $116(54.2)$ & 0.55 \\
\hline \multicolumn{5}{|l|}{ Comorbidities } \\
\hline Hypertension & $193(32.7)$ & $126(33.4)$ & 67 (31.3) & 0.60 \\
\hline Diabetes & $132(22.3)$ & 73 (19.4) & $59(27.6)$ & 0.02 \\
\hline Cardiovascular disease & $65(11.0)$ & 45 (11.9) & $20(9.4)$ & 0.33 \\
\hline Chronic lung disease & $35(5.9)$ & $25(6.6)$ & $10(4.7)$ & 0.33 \\
\hline Chronic renal disease & $27(4.6)$ & $15(4.0)$ & $12(5.6)$ & 0.36 \\
\hline Chronic hepatic disease & $60(10.2)$ & $35(9.3)$ & $25(11.7)$ & 0.35 \\
\hline Malignancy & $339(57.4)$ & $221(58.6)$ & $118(55.1)$ & 0.41 \\
\hline Neutropenia & $100(16.9)$ & 75 (19.9) & $25(11.7)$ & 0.10 \\
\hline Suspected infection site & & & & 0.54 \\
\hline Intra-abdominal infection & $229(38.8)$ & $138(36.6)$ & $91(42.5)$ & 0.16 \\
\hline Pneumonia & $166(28.1)$ & $108(28.7)$ & $58(27.1)$ & 0.69 \\
\hline Urinary tract infection & $94(15.9)$ & 63 (16.7) & $31(14.5)$ & 0.48 \\
\hline Others & $102(17.3)$ & 68 (18.0) & $34(15.9)$ & 0.51 \\
\hline \multicolumn{5}{|l|}{ Initial vital signs } \\
\hline MAP (mmHg) & $71(60-84)$ & $71(58-83)$ & $71(62-86)$ & 0.19 \\
\hline Heart rate (/min) & $114(97-132)$ & $114(98-133)$ & $114(95-130)$ & 0.37 \\
\hline Respiratory rate (/min ) & $20(20-24)$ & $20(20-24)$ & $20(20-24)$ & 0.64 \\
\hline Body temperature $\left({ }^{\circ} \mathrm{C}\right)$ & 38.0 (36.8-38.9) & $38.2(37.0-39.0)$ & $37.2(36.6-38.6)$ & $<0.01$ \\
\hline Positive blood culture & $233(39.4)$ & $151(40.1)$ & $82(38.3)$ & 0.68 \\
\hline Initial presentation with hypotension & $247(41.8)$ & $181(48.0)$ & 66 (30.8) & $<0.01$ \\
\hline Initial serum lactate (mmol/L) & $4.8(4.1-6.4)$ & $4.8(3.9-6.4)$ & $5.0(4.2-6.2)$ & 0.27 \\
\hline APACHE II score & $15(10-19)$ & $15(11-20)$ & $14(10-18)$ & 0.03 \\
\hline Use of vasopressors within $24 \mathrm{hr}$ & $364(61.6)$ & $244(64.7)$ & $120(56.1)$ & 0.04 \\
\hline Mechanical ventilation & $78(13.2)$ & $47(12.5)$ & $31(14.5)$ & 0.49 \\
\hline \multicolumn{5}{|l|}{ Targets of initial resuscitation } \\
\hline CVP $\geq 8 \mathrm{mmHg}$ achieved & $301(50.9)$ & $206(54.6)$ & $95(44.4)$ & 0.02 \\
\hline $\mathrm{MAP} \geq 65 \mathrm{mmHg}$ achieved & $582(98.5)$ & $372(98.7)$ & $210(98.1)$ & 0.60 \\
\hline $\mathrm{ScvO}_{2} \geq 70 \%$ achieved & $296(50.1)$ & $206(54.6)$ & $90(42.1)$ & $<0.01$ \\
\hline
\end{tabular}

Values are presented as median (interquartile range) or number (\%).

APACHE, Acute Physiology and Chronic Health Evaluation; CVP, central venous pressure; MAP, mean arterial pressure; ScvO ${ }_{2}$ central venous oxygen saturation. 
Table 2. Comparison of outcomes including in-hospital mortality, delta SOFA, and length of hospital stay

\begin{tabular}{lcccr}
\hline Variable & $\begin{array}{c}\text { All patients } \\
(\mathrm{n}=591)\end{array}$ & $\begin{array}{c}\text { Early group } \\
(\mathrm{n}=377)\end{array}$ & $\begin{array}{c}\text { Delayed group } \\
(\mathrm{n}=214)\end{array}$ & P-value \\
\hline $\begin{array}{l}\text { In-hospital mortality } \\
\text { SOFA score }\end{array}$ & $110(18.6)$ & $61(16.2)$ & $49(22.9)$ & 0.04 \\
$\quad$ Baseline & $7(4-9)$ & $7(4-10)$ & $6.5(3-9)$ & 0.01 \\
$\quad 48$ hours & $4(2-7)$ & $4(2-7)$ & $4(1-8)$ & 0.61 \\
$\quad$ Delta SOFA & $2(0-4)$ & $2(0-5)$ & $1(-1$ to 3$)$ & $<0.01$ \\
In-hospital LOS (day) & & & & \\
$\quad$ All patients & $12(7-22)$ & $11(7-21)$ & $14.5(8-24)$ & $<0.01$ \\
$\quad$ Survivors only & $12(8-22)$ & $11(8-22)$ & $15(9-23)$ & $<0.01$ \\
ICU admission & $303(51.3)$ & $190(50.4)$ & $113(52.8)$ & 0.57 \\
LOS in ICU (day) & $3(2-7)$ & $3(2-6)$ & $3(2-8)$ & 0.12 \\
\hline
\end{tabular}

Values are presented as number (\%) or median (interquartile range).

SOFA, Sequential Organ Failure Assessment; LOS, length of stay; ICU, intensive care unit.

214 patients (36.2\%) had delayed administration. Median time to antibiotic use was 1.9 hours (IQR, 1.4 to 2.4 hours) in the early administration group and 4.4 hours (IQR, 3.5 to 6.8 hours) in the delayed administration group. In the delayed administration group, diabetes was more frequent, and initial body temperature and APACHE II score were significantly lower. In the early administration group, initial presentation with hypotension, use of vasopressors, and achievement of early resuscitation targets (CVP and $\mathrm{ScvO}_{2}$ ) were more frequent.

\section{Comparison of outcomes}

The in-hospital mortality rate was $16.2 \%$ for the early administration group and $22.9 \%$ for the delayed administration group, with a significant difference $(P=0.04)$ (Table 2$)$. The early administration group had a significantly higher SOFA score at the time of diagnosis, but there was no significant difference in SOFA scores after 48 hours. Delta SOFA turned out to be significantly higher in the early administration group $(P<0.01)$. Length of hospital stay was significantly shorter in patients with early administration group for both the entire cohort and the surviving patient group $(\mathrm{P}<0.01)$. ICU admission rates and ICU LOS were not significantly different between groups.

\section{Multivariable logistic regression analysis for in-hospital mortality}

In multivariable logistic regression analysis adjusted for confounding factors, early administration of antibiotics was independently associated with reduction of the in-hospital mortality rate (adjusted odds ratio [OR], 0.54; 95\% confidence interval [Cl], 0.34 to $0.87 ; \mathrm{P}=0.01$ ) (Table 3 ).
Table 3. Multivariable logistic regression analysis to identify independent predictors of in-hospital mortality

\begin{tabular}{lccc}
\hline Variable & Adjusted OR & $95 \% \mathrm{Cl}$ & P-value \\
\hline Timely antibiotic use & 0.54 & $0.34-0.87$ & 0.01 \\
APACHE II score & 1.06 & $1.03-1.10$ & $<0.01$ \\
Infection focus & & & \\
$\quad$ Abdomen & Reference & & \\
$\quad$ Pneumonia & 2.31 & $1.34-3.94$ & $<0.01$ \\
$\quad$ Urinary tract infection & 0.19 & $0.52-0.66$ & $<0.01$ \\
$\quad$ Others & 1.76 & $0.93-3.33$ & 0.08 \\
Initial serum lactate (mmol/L) & 1.20 & $1.03-1.10$ & $<0.01$ \\
\hline
\end{tabular}

Hosmer-Lemeshow goodness-of-fit test, $\mathrm{P}=0.83$.

$\mathrm{OR}$, odds ratio; $\mathrm{Cl}$, confidence interval; $\mathrm{APACHE}$, Acute Physiology and Chronic Health Evaluation.

Table 4. Multivariable linear regression analysis for the delta SOFA and in-hospital length of stay

\begin{tabular}{lccr}
\hline Variable & $\begin{array}{c}\text { Regression } \\
\text { coefficient }\end{array}$ & $\begin{array}{c}\text { Standard } \\
\text { error }\end{array}$ & P-value \\
\hline Analysis for the delta SOFA & & & \\
$\quad$ Timely antibiotic use & 0.73 & 0.36 & 0.04 \\
APACHE II score & 0.06 & 0.02 & $<0.01$ \\
Initial serum lactate & -0.29 & 0.06 & $<0.01$ \\
Scv0 2 70\% achieved & 1.20 & 0.35 & $<0.01$ \\
Analysis for in-hospital LOS & & & \\
$\quad$ Timely antibiotic use & -0.28 & 0.08 & $<0.01$ \\
APACHE II score & 0.01 & 0.01 & 0.06 \\
Chronic renal disease & 0.36 & 0.18 & 0.05 \\
Chronic hepatic disease & 0.39 & 0.13 & $<0.01$ \\
Malignancy & 0.22 & 0.08 & 0.01 \\
CVP $\geq 8$ mmHg achieved & 0.19 & 0.08 & 0.02 \\
\hline
\end{tabular}

SOFA, Sequential Organ Failure Assessment; APACHE, Acute Physiology and Chronic Health Evaluation; $\mathrm{ScvO}_{2}$, central venous oxygen saturation; LOS, length of stay; CVP, central venous pressure.

${ }^{a}$ The In-hospital LOS values were log transformed in order to obtain a normal distribution.

\section{Multivariable linear regression analysis for delta SOFA and in-hospital LOS}

In multivariable linear regression analysis on delta SOFA, early administration of antibiotics was significantly associated with an increase in the delta SOFA, which is evidence that early administration of antibiotics is related to recovery from organ failure (coefficient, 0.73; standard error, 0.36; $P=0.04$ ) (Table 4). Delayed administration was significantly associated with prolongation of length of hospitalization, whereas early administration reduced the length of hospitalization by $28 \%$ compared with delayed administration.

\section{DISCUSSION}

This study analyzed the effect of timely administration of a broadspectrum antibiotics to patients with severe sepsis and septic shock, 
as recommended by the 2012 SSC guidelines, on prognostic factors, including in-hospital mortality rate, level of recovery from organ failure, and the length of hospitalization. In this study, early administration of antibiotics was significantly associated with survival to hospital discharge and also had significant associations with reduction of 48-hour SOFA scores and reduced hospital LOS.

The correlation between timely administration of antibiotics and mortality rates among infected patients has been previously studied in community-dwelling patients with pneumonia, and McGarvey and Harper ${ }^{17}$ reported that for community-dwelling patients with pneumonia, administration of antibiotics within 4 hours was significantly associated with a reduction in the mortality rate. Kumar et al. ${ }^{8}$ reported that in cases of septic shock, every one-hour delay in antibiotic administration from the time when low blood pressure was first apparent significantly increased mortality rates (adjusted OR, 1.119 [per hour delay]; 95\% Cl, 1.103 to 1.136$)$. Most recently, Ferrer et al. ${ }^{6}$ reported in a retrospective study on patients with severe sepsis and septic shock in 165 ICUs that the time of antibiotic administration had a quantitative relationship with mortality rate. The results of our study correspond with observational studies that have reported a relationship between the timing of antibiotic administration and mortality rates, which supports the clinical importance of prompt administration of antibiotics as recommended by the 2012 SSC guidelines.

This study demonstrated that delayed administration of antibiotics for patients with severe sepsis and septic shock might worsen organ dysfunction or reduce the level of recovery of organs, which may consequently extend the length of treatment. Our results were adjusted for severity and achievement of early resuscitation targets, and demonstrated that early administration of antibiotics is an important qualitative index in the treatment of patients with severe sepsis and septic shock. In particular, it is noteworthy that although the group with early administration had a relatively high level of severity, the early administration group had a better prognosis. These results imply that delayed administration of antibiotics might not just increase the mortality rate but also raise the risk of complications during treatment, extend the length of hospitalization, and lead to increased medical costs.

Multiple steps, such as the initial examination, blood tests, diagnosis of sepsis, blood cultures, and prescription of antibiotics, are required before the actual administration of antibiotics, which makes timely administration difficult. ${ }^{10} \mathrm{As}$ a result, the rate of patients with severe sepsis and septic shock with delayed administration of antibiotics remains too high and continual efforts to improve are necessary. ${ }^{6,18}$ In our study, according to the differ- ences between early and delayed administration groups, there might be potential barriers for early administration including diabetics, lower initial body temperature, and cryptic shock without hypotension. Those factors might influence the physician's recognition of sepsis and the initiation of early resuscitation, potentially resulting in the poor outcomes. We suggest that it is especially critical to make efforts to prevent delayed administration of antibiotics for these patient groups through rapid diagnosis of severe sepsis.

It is not only important that antibiotic administration be prompt, but that the antibiotics used in the early stages of sepsis are appropriate for the causative pathogens. ${ }^{19}$ Kumar et al. ${ }^{20}$ reported that among 5,715 patients with septic shock, around 20\% of patients were administered inappropriate antibiotics, which increased the mortality rate five times relative to cases with appropriate administration of antibiotics. Our study did not analyze the relationship between appropriate administration of antibiotics for causative pathogens and prognosis, which is the main limitation of this study. However, we evaluated the time to administration of broad-spectrum antibiotics covering most of the possible pathogens. Therefore, our results do not support that the use of early inappropriate antibiotics is beneficial.

Our study was a retrospective observational study and had the following limitations. First, as a study of a single institution, the results of the study cannot be generalized to other institutions. Second, with a small study population, the administration time of antibiotics was not specifically analyzed, and we were unable to evaluate for a linear relationship between prognosis and time of antibiotic use, and we did not determine the ideal administration time for antibiotics. Third, although this study observed a relationship between appropriate timing of antibiotics and recovery from organ failure and length of hospitalization, we were unable to evaluate the mechanism by which early administration of antibiotics affects the recovery of organ failure and length of hospitalization. Additional studies are required to evaluate organ failure in detail. Lastly, although this study used a prospectively collected registry, additional data were collected through medical records. In conclusion, early administration of antibiotics within three hours from ED arrival for patients with severe sepsis or septic shock was significantly associated with reduced in-hospital mortality, recovery of organ dysfunction, and decreased hospital LOS.

\section{CONFLICT OF INTEREST}

No potential conflict of interest relevant to this article was reported. 


\section{REFERENCES}

1. Bone RC, Balk RA, Cerra FB, et al. Definitions for sepsis and organ failure and guidelines for the use of innovative therapies in sepsis. The ACCP/SCCM Consensus Conference Committee. American College of Chest Physicians/Society of Critical Care Medicine. Chest 1992;101:1644-55.

2. Levy MM, Fink MP, Marshall JC, et al. 2001 SCCM/ESICM/ ACCP/ATS/SIS International Sepsis Definitions Conference. Crit Care Med 2003;31:1250-6.

3. Martin GS, Mannino DM, Eaton S, Moss M. The epidemiology of sepsis in the United States from 1979 through 2000. N Engl J Med 2003;348:1546-54.

4. Angus DC, Linde-Zwirble WT, Lidicker J, Clermont G, Carcillo J, Pinsky MR. Epidemiology of severe sepsis in the United States: analysis of incidence, outcome, and associated costs of care. Crit Care Med 2001;29:1303-10.

5. Khwannimit B, Bhurayanontachai R. The epidemiology of, and risk factors for, mortality from severe sepsis and septic shock in a tertiary-care university hospital setting. Epidemiol Infect 2009;137:1333-41.

6. Ferrer R, Martin-Loeches I, Phillips G, et al. Empiric antibiotic treatment reduces mortality in severe sepsis and septic shock from the first hour: results from a guideline-based performance improvement program. Crit Care Med 2014;42:1749-55.

7. Puskarich MA, Trzeciak S, Shapiro NI, et al. Association between timing of antibiotic administration and mortality from septic shock in patients treated with a quantitative resuscitation protocol. Crit Care Med 2011;39:2066-71.

8. Kumar A, Roberts D, Wood KE, et al. Duration of hypotension before initiation of effective antimicrobial therapy is the critical determinant of survival in human septic shock. Crit Care Med 2006;34:1589-96.

9. Gaieski DF, Mikkelsen ME, Band RA, et al. Impact of time to antibiotics on survival in patients with severe sepsis or septic shock in whom early goal-directed therapy was initiated in the emergency department. Crit Care Med 2010;38:1045-53.
10. Dellinger RP, Levy MM, Rhodes $A$, et al. Surviving Sepsis Campaign: international guidelines for management of severe sepsis and septic shock: 2012. Crit Care Med 2013;41:580-637.

11. Dellinger RP, Levy MM, Carlet JM, et al. Surviving Sepsis Campaign: international guidelines for management of severe sepsis and septic shock: 2008. Crit Care Med 2008;36:296-327.

12. Kang MJ, Shin TG, Jo IJ, et al. Factors influencing compliance with early resuscitation bundle in the management of severe sepsis and septic shock. Shock 2012;38:474-9.

13. Rivers E, Nguyen B, Havstad S, et al. Early goal-directed therapy in the treatment of severe sepsis and septic shock. N Engl J Med 2001;345:1368-77.

14. Zimmerman JE, Kramer AA, McNair DS, Malila FM. Acute Physiology and Chronic Health Evaluation (APACHE) IV: hospital mortality assessment for today's critically ill patients. Crit Care Med 2006;34:1297-310.

15. Ferreira FL, Bota DP, Bross A, Melot C, Vincent JL. Serial evaluation of the SOFA score to predict outcome in critically ill patients. JAMA 2001;286:1754-8.

16. Vincent JL, Moreno R, Takala J, et al. The SOFA (Sepsis-related Organ Failure Assessment) score to describe organ dysfunction/failure. On behalf of the Working Group on Sepsis-Related Problems of the European Society of Intensive Care Medicine. Intensive Care Med 1996;22:707-10.

17. McGarvey RN, Harper JJ. Pneumonia mortality reduction and quality improvement in a community hospital. QRB Qual Rev Bull 1993;19:124-30.

18. De Miguel-Yanes JM, Andueza-Lillo JA, Gonzalez-Ramallo VJ, Pastor L, Munoz J. Failure to implement evidence-based clinical guidelines for sepsis at the ED. Am J Emerg Med 2006;24: 553-9.

19. Barochia AV, Cui X, Vitberg D, et al. Bundled care for septic shock: an analysis of clinical trials. Crit Care Med 2010;38:668-78.

20. Kumar A, Ellis $P$, Arabi Y, et al. Initiation of inappropriate antimicrobial therapy results in a fivefold reduction of survival in human septic shock. Chest 2009;136:1237-48. 